\title{
Variabilité spatio-temporelle des apports liquide et solide en zone semi-aride. Cas du bassin versant de l'oued Mina (nord-ouest algérien) Spatial and temporal variability of streamflow and solid yields in semiarid areas. Case of the oued Mina basin (Northwest Algeria)
}

\section{Achite et M. Meddi}

Volume 18, numéro hors-série, 2005

URI : https://id.erudit.org/iderudit/705575ar

DOI : https://doi.org/10.7202/705575ar

\section{Aller au sommaire du numéro}

\section{Éditeur(s)}

Université du Québec - INRS-Eau, Terre et Environnement (INRS-ETE)

\section{ISSN}

0992-7158 (imprimé)

1718-8598 (numérique)

\section{Découvrir la revue}

Citer cet article

Achite, M. \& Meddi, M. (2005). Variabilité spatio-temporelle des apports liquide et solide en zone semi-aride. Cas du bassin versant de l'oued Mina (nord-ouest algérien). Revue des sciences de l'eau / Journal of Water Science, 18, 37-56. https://doi.org/10.7202/705575ar

\section{Résumé de l'article}

Les zones méditerranéennes et semi - arides sont caractérisées par des régimes pluviométriques irréguliers dans le temps et dans l'espace. L'érosion hydrique des bassins versants et la sédimentation des cours d'eau constituent des phénomènes complexes très répandus qui posent de nombreux problèmes aux ingénieurs chargés des projets de mise en valeur. En raison de son ampleur, cette érosion constitue une contrainte majeure au développement de l'agriculture et à la promotion des activités rurales en Algérie. Une approche statistique de quantification des apports solides est développée dans cette étude. Les données utilisées sont recueillies au niveau de cinq sous-bassins du bassin versant de l'Oued Mina drainés par les stations hydrométriques, en amont du barrage de Sidi M'hamed Ben Aouda. La période d'observation s'étale sur 22 ans (1973 à 1995). Il existe une grande variabilité inter-annuelle et intrannuelle des apports liquides et solides. En plus de cette variation temporelle, il a été remarqué une variabilité spatiale des apports solides et liquides.

Les valeurs maximales du transport solide sont enregistrées au début d'automne et à la fin du printemps. Le transport solide d'automne reste le plus élevé, dépassant significativement les autres saisons. Cette variabilité trouve son explication dans la pauvreté du couvert végétal durant cette saison et la nature agressive des pluies d'automne. La charge spécifique du bassin versant de l'oued Haddad est de l'ordre de $212 \mathrm{t} / \mathrm{km}^{2} / \mathrm{an}$. Le bassin versant de l'oued Taht a une charge spécifique inférieure à celle du bassin de l'oued Haddad (Ds=191 t $/ \mathrm{km}^{2} / \mathrm{an}$ ). Les bassins versants de l'oued el Abd présentent des charges spécifiques de l'ordre de $117 \mathrm{t} / \mathrm{km}^{2} / \mathrm{an}$ à Ain El Hamara et de $65 \mathrm{t} / \mathrm{km}^{2} / \mathrm{an}$ à Takhmaret. La charge spécifique de l'oued Mina au barrage de Sidi M'hamed Ben Aouda est de $160 \mathrm{t} / \mathrm{km}^{2} / \mathrm{an}$.

Des modèles mathématiques liant l'apport solide au coefficient d'écoulement ont été proposés pour les cinq sous-bassins drainés par les stations hydrométriques. 


\title{
Variabilité spatio-temporelle des apports liquide et solide en zone semi-aride. Cas du bassin versant de l'oued Mina (nord-ouest algérien)
}

\author{
Spatial and temporal variability of streamflow and \\ solid yields in semiarid areas. Case of the oued Mina \\ basin (Northwest Algeria)
}

\author{
M. ACHITE ${ }^{1 *}, M . M E D D I^{2}$
}

Reçu le 19 décembre 2003, accepté le 17 février 2005**.

\section{SUMMARY}

Mediterranean and semi-arid zones are characterized by an aggressive climate and by irregular and violent rainfall events. Water-induced erosion in river basins and river sedimentation constitute a complex phenomenon, which poses many problems for the engineers responsible for development projects. This phenomenon constitutes a major constraint limiting agricultural development in Algeria. We propose a statistical approach to quantify the solid yield at different gauging sites.

The data were collected at five sites in the oued Mina basin (upstream of the Sidi Me hamed Ben Aouda dam). The observation period covered 22 years, from 1973 to 1995 . The results showed a high inter-annual and annual variability of the liquid discharge and solid yields; the coefficient of variation exceeded $60 \%$ in all cases. In addition to this temporal variation, we noted a variability of the solid yields and liquid discharge among the different sites. Analysis of the monthly solid yields shows that the autumn season contributes a large proportion of the annual solid yield (more than $40 \%$ ) for the different basins. Tranport of solids in the oued Mina and its effluents is highly variable in space and time. This variability is due primarily to the pluviometric regime, the vegetation cover and the lithology. In general, the seasonal variations of the solid contributions follow those of the liquid flow. The maximum values of the solid discharges, for all basins, are observed at the beginning of autumn and the end of spring. Generally, the monthly minimal solid yields are recorded in summer. This season is characterized by low rainfall and consequently a weak surface flow. However, the minimal solid yields do not necessarily correspond to the month characterized by the lowest liquid discharge. The autumn contributes $31 \%$ to the annual liquid flow and

1. Université des Sciences et de la Technologie d'Oran, Faculté d'Architecture et de Génie Civil, Département d'Hydraulique, B.P. 1505 El M'naouar, 31000, Oran, tél./fax : 041425875.

2. LRERP, Centre Universitaire de Khemis Miliana, 44225 Khemis Miliana, tél/fax : 027664863.

* Correspondance: achite_meddz@yahoo.fr, mmeddi@yahoo.fr

** Les commentaires seront reçus jusqu'au 31 mai 2006. 
$51 \%$ to the annual solid yields of the oued Mina area at Wadi El Abtal station; corresponding values are $(26 \%, 33 \%),(33 \%, 53 \%),(29 \%, 46 \%)$ and $(36 \%, \mathbf{4 8 \%})$ at the stations of Sidi A.E.K Djillali, Ain Hamara, Kef Mehboula and Takhmaret, respectively. The autumn solid transport remains highest, significantly exceeding the other seasons. This variability is explained by the variation in vegetation cover (bare ground) during the year and the aggressive nature of the autumn rains. Because of these two factors, the first autumn flood transports considerable quantities of sediments after a long dry season characterized by high temperatures and destruction of the soil aggregates by these temperatures. The energy produced by the first rains and the low degree of vegetative cover rate in this season also contribute to this increase in autumn. The oued Haddad basin presents a high specific degradation compared to the other basins (Ds $=212 \mathrm{t} / \mathrm{km}^{2} / \mathrm{an}$ ). This impoverishment of the soil is very visible at this site. It is due to factors favouring erosion: steep topography and discontinuous vegetative cover, which protects the ground poorly. The oued That basin, which has a topography favouring surface flow and abundant rains, has a specific degradation lower than the oued Haddad basin (Ds $=191 \mathrm{t} / \mathrm{km}^{2} / \mathrm{an}$ ). The basins of oued El Abd present a specific degradation about $117 \mathrm{t} / \mathrm{km}^{2} / \mathrm{an}$ at Ain El Hamara station and about $65 \mathrm{t} / \mathrm{km}^{2} /$ an at Takhmaret station. The oued Mina at Sidi M'hamed Ben Aouda dam gives a specific degradation about $187 \mathrm{t} / \mathrm{km}^{2} / \mathrm{an}$.

The runoff coefficient explains a large part of the variance of the solid yield (more than $60 \%$ ). This coefficient depends intrinsically on lithology, geology, vegetative cover, topography and the hydrological and pluviometric regimes. By the introduction of this parameter, we sought to explain solid transport by the explanatory variables of this phenomenon. The result was very encouraging, considering the percentage of the explained variance (more than $60 \%$ ). Following this analysis, mathematical models are proposed, connecting the solid yield to the runoff coefficient. The determinations coefficients are equal to: $0.79 ; 0.61 ; 0.64 ; 0.78 ; 0.65$ for the stations of oued Al Abtal, Sidi Abdelkader Djillali, Ain Hamara, Kef Mehboula and Takhmaret, respectively.

Keywords: liquid discharge, solid yield, erosion, spatial and temporal variability, statistical model, oued Mina, Algeria.

\section{RÉSUMÉ}

Les zones méditerranéennes et semi-arides sont caractérisées par des régimes pluviométriques irréguliers dans le temps et dans l'espace. L'érosion hydrique des bassins versants et la sédimentation des cours d'eau constituent des phénomènes complexes très répandus qui posent de nombreux problèmes aux ingénieurs chargés des projets de mise en valeur. En raison de son ampleur, cette érosion constitue une contrainte majeure au développement de l'agriculture et à la promotion des activités rurales en Algérie. Une approche statistique de quantification des apports solides est développée dans cette étude. Les données utilisées sont recueillies au niveau de cinq sousbassins du bassin versant de l'oued Mina drainés par les stations hydrométriques, en amont du barrage de Sidi M'hamed Ben Aouda. La période d'observation s'étale sur 22 ans (1973 à 1995). Il existe une grande variabilité inter-annuelle et intra- annuelle des apports liquide et solide. En plus de cette variation temporelle, il a été remarqué une variabilité spatiale des apports solides et liquides.

Les valeurs maximales du transport solide sont enregistrées au début d'automne et à la fin du printemps. Le transport solide d'automne reste le plus élevé, dépassant significativement les autres saisons. Cette variabilité trouve son explication dans la pauvreté du couvert végétal durant cette saison et la nature agressive des pluies d'automne. La charge spécifique du bassin ver- 
sant de l'oued Haddad est de l'ordre de $212 \mathrm{t} / \mathrm{km}^{2} / \mathrm{an}$. Le bassin versant de l'oued Taht a une charge spécifique inférieure à celle du bassin de l'oued Haddad (Ds $=191 \mathrm{t} / \mathrm{km}^{2} / \mathrm{an}$ ). Les bassins versants de l'oued el Abd présentent des charges spécifiques de l'ordre de $117 \mathrm{t} / \mathrm{km}^{2} / \mathrm{an}$ à Ain El Hamara et de $65 \mathrm{t} / \mathrm{km}^{2} / \mathrm{an}$ à Takhmaret. La charge spécifique de l'oued Mina au barrage de Sidi M'hamed Ben Aouda est de $187 \mathrm{t} / \mathrm{km}^{2} / \mathrm{an}$.

Des modèles mathématiques liant l'apport solide au coefficient d'écoulement ont été proposés pour les cinq sous-bassins drainés par les stations hydrométriques.

Mots clés : apport liquide, apport solide, érosion, variabilité spatio-temporelle, modèle statistique, oued Mina, Algérie.

\section{1 - INTRODUCTION}

Dans les zones semi-arides, caractérisées par des précipitations très irrégulières souvent intenses, les facteurs climatiques ont une influence considérable sur les pertes en sol (BERGAOUI et al., 1998). Or, en région méditerranéenne, la quantification des matières transportées par les cours d'eau est une entreprise peu aisée, du fait du caractère irrégulier du régime hydrologique (SERRAT et al., 2001) et du manque de données nécessaires à ces études. L'érosion et le transport solide constituent une contrainte majeure au développement de l'agriculture et à la gestion des ouvrages hydrotechniques (envasés à plus de $20 \%$ ) en Algérie.

Les travaux relatifs à la modélisation des phénomènes d'érosion et du transport solide ont mis au point des relations liant le transport solide aux paramètres explicatifs (ex : le débit liquide, la pluie, l'humidité du sol). Cette entreprise est indispensable pour permettre de comprendre l'évolution spatio-temporelle du phénomène et de quantifier les charges solides aux exutoires des bassins versants. En Algérie, comme dans les autres pays méditerranéens, de nombreux modèles ont été mis au point $(\mathrm{HEUSCH}, 1970$, DELHOUME, 1981, DEMMAK, 1982 et 1984, BERGAOUI et al. 1998, LAHLOU, 1994, KASSOUL et al., 1997, BENKHALED et REMINI, 2003, TERFOUs et al. 2001, MEDDI et al., 1998, MEDDI, 1999, MEDDI et MORSLI, 2001, ACHITE et TOUAIBIA, 2000, ACHITE, 2002, BOU KHEIR et al., 2001). Des modèles plus généraux ont été développés sous d'autres climats (WALLING et WEBB, 1981a,b), FERGUSON R.I., 1987). L'objectif est de développer une expression explicative du transport solide par le débit liquide. Ce dernier servira à quantifier le transport solide en utilisant l'information sur le débit liquide qui est plus abondante (à l'échelle journalière) que celle sur transport solide. Dans un deuxième temps, des modèles liant le transport solide au coefficient de ruissellement seront développés. Ils tiennent compte de la pluviométrie, du ruissellement et de l'état de l'humidité du sol. Ces modèles peuvent servir comme moyen de prévision du transport solide pour les différents bassins. 


\section{2 - MATÉRIEL ET MÉTHODES}

\subsection{Le bassin versant de l'oued Mina}

Le bassin versant de l'oued Mina fait partie du plus grand bassin versant de l'Algérie du Nord à savoir l'oued Chellif (figure 1). II se trouve à environ $300 \mathrm{~km}$ à l'Ouest d'Alger, entre $0^{\circ} 20^{\prime}$ et $1^{\circ} 10^{\prime}$ ' de longitude Est et entre $34^{\circ} 40^{\prime}$ et $35^{\circ} 40^{\prime}$ de latitude Nord. II draine une superficie de 4900 km² jusqu'au barrage Sidi M'Hamed Ben Aouda. L'oued Mina compte parmi les principaux affluents de l'oued Cheliff. II parcourt une distance de $135 \mathrm{~km}$ environ entre les barrages de Bakhada et de Sidi M'hamed Ben Aouda, avec une orientation Sud-Est, Nord-Ouest. L'étude topographique (MEDDI, 1992) a permis de ranger les bassins étudiés dans la classe de relief fort, selon la classification de l'ORSTOM (DUBREUIL et GUSCAFRE, 1971). À partir des courbes hypsométriques et des courbes des fréquences altimétriques (MEDDI, 1992), on peut tirer les conclusions suivantes: les tranches d'altitude supérieures à $1200 \mathrm{~m}$ sont très peu étendues dans le bassin versant de l'oued Mina, 4,14\% de surface dans le bassin versant de l'oued el Abd à Takhmaret. Au niveau du bassin versant de l'oued Haddad, 0,16\% de la surface est représentée par des altitudes supérieures à $1100 \mathrm{~m}$. Les zones d'altitude couvrent $4,23 \%$ de la superficie du bassin versant de l'oued Tat. Pour le bassin versant de l'oued Mina à l'oued el Abtal, 1,6\% de la superficie est représentée par des altitudes supérieures à $1200 \mathrm{~m}$.

Le climat de la région est de type semi-aride méditerranéen (pluies en hiver, sécheresse en été), avec une précipitation moyenne annuelle estimée à $305 \mathrm{~mm}$ (ACHITE, 1999). La majorité des précipitations se concentre entre novembre et mars. Du point de vue géologique, le bassin versant de l'oued Mina est caractérisé par la prépondérance du jurassique au nord et à l'ouest. Le reste du bassin présente un paysage crétacé avec des roches calcaires et de grès. Par contre, le centre et l'ouest de la partie septentrionale sont soumis au phénomène érosif. Le bassin versant de l'oued Haddad draine des marnes sensibles à l'érosion hydrique et l'oued el Abd draine une zone de calcaires et de marnes subordonnées. Sur les Monts de Frenda et de Saida au sud, les calcaires affleurent souvent.

L'analyse de la diversité et de la répartition géographique des formations végétales sur le bassin versant de l'oued Mina se présente en deux zones nettement distinctes (KOURI, 1993 et MAHIEDDINE, 1997), à savoir :

- La zone nord, purement marneuse, est fortement érodée et dépourvue de végétation dans la plupart des terres à l'exception des quelques îlots de reboisement de Tassaalat et des plantations d'arbres fruitiers dans la vallée. Les sols de cette partie du bassin versant sont généralement nus, surtout sur les terrains rocheux. La discontinuité ou l'absence totale de la végétation, l'ampleur morphodynamique due à la faible résistance des terrains et de leurs pentes favorisent l'érosion.

- La zone jurassique du sud est moins érodée et près de $50 \%$ de sa surface est recouverte par une végétation de densité variable. Sur cette zone les forêts représentent $5,8 \%$ de la partie sud du bassin versant. Elles sont dominées par une grande partie de jeunes plantations de pin d'Alep. Les maquis représentent environ $32 \%$ de l'ensemble du bassin versant. Ils 
existent sous deux formes: maquis avec PISTACIA et OLEA et maquis avec TETRACLINIS. La végétation herbacée est représentée, en plus de la végétation naturelle, par les céréales qui sont les principales cultures annuelles.

\subsection{Collecte et mise en forme des données}

\subsubsection{Réseau pluviométrique}

Parmi toutes les stations pluviométriques disponibles, nous avons retenu uniquement celles qui présentaient une période de mesure longue et commune, soit de 1973 à 1995 (tableau 1 et figure 1).

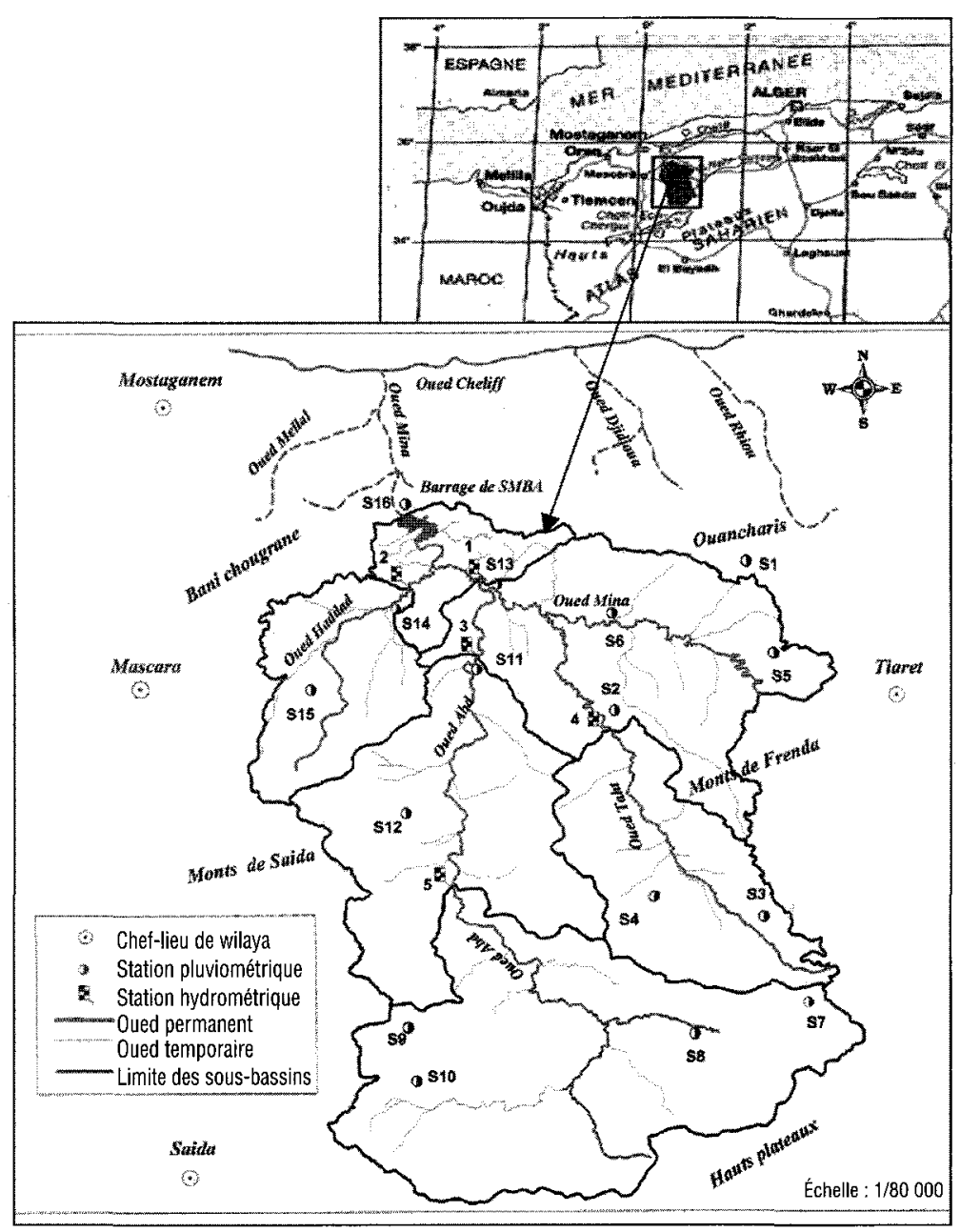

Figure 1 Situation du bassin versant de l'Oued Mina.

Geographical location of the Oued Mina Basin. 
Tableau 1 Stations pluviométriques du bassin versant de l'oued Mina.

Table 1 Precipitation stations in the wadi Mina basin.

\begin{tabular}{lcccr}
\hline $\mathbf{N}^{\circ}$ & Station & $\mathbf{X}(\mathbf{K m})$ & $\mathbf{Y}(\mathbf{K m})$ & $\mathbf{Z}(\mathbf{m})$ \\
\hline S1 & Rahuia & 347,85 & 249,15 & 650 \\
S2 & Kef-Mehboula & 331,60 & 224,25 & 475 \\
S3 & Frenda & 348,60 & 197,00 & 990 \\
S4 & Ain El Haddid & 334,50 & 197,00 & 829 \\
S5 & Mechra safa & 351,00 & 233,20 & 655 \\
S6 & Djilali Benamar & 232,50 & 239,90 & 300 \\
S7 & Ain Kermes & 354,55 & 179,85 & 1162 \\
S8 & Rosfa & 330,60 & 179,50 & 960 \\
S9 & Tiricine & 303,90 & 180,50 & 1070 \\
S10 & Sidi Youcef & 305,60 & 169,10 & 1100 \\
S11 & Ain Hamara & 316,45 & 233,12 & 288 \\
S12 & Takmaret & 316,40 & 203,10 & 655 \\
S13 & oued El Abtal & 318,05 & 261,70 & 354 \\
S14 & Sidi A.E.K Djilali & 309,00 & 244,50 & 225 \\
S15 & El Hachem & 299,50 & 233,10 & 417 \\
S16 & Sidi M'Hamed Ben Aouda & 308,85 & 255,18 & 145 \\
\hline
\end{tabular}

avec $\mathrm{X}$ : Longitude en $\mathrm{km}, \mathrm{Y}$ : Latitude en $\mathrm{km}$ et $\mathrm{Z}$ : Altitude.

\subsubsection{Réseau hydrométrique et mesure des matières en suspension}

La mesure de la concentration de matières en suspension consiste en des prises systématiques d'échantillons d'eau à l'aide d'un flacon d'une contenance de $500 \mathrm{ml}$. Ces échantillons sont pris en un point unique de l'oued, soit sur le bord ou soit au milieu. Les prélèvements sont plus nombreux en période de crue, alors qu'en étiage ou lorsque le débit liquide est constant au cours de la journée, un seul prélèvement est effectué par jour. La difficulté de la mesure est la variabilité de la concentration en sédiment dans la section de mesure. La mesure du débit liquide est faite à partir de stations hydrométriques fixes.

Dans tous les cas, il s'avérait essentiel de collecter un nombre suffisant de données fiables afin de pouvoir estimer les apports solides et permettre le calage des modèles proposés.

Les données de base disponibles sont:

- Débits liquides instantanés $\left(\mathrm{m}^{3} / \mathrm{s}\right)$;

- Concentrations correspondantes aux débits liquides instantanés $C$ (en $\mathrm{g} / \mathrm{l}$ ), obtenues à partir des fiches d'analyse des eaux.

Les données d'observations ont été mises sous forme d'un fichier contenant le numéro de l'observation, la date, l'heure, la hauteur d'eau, la concentration, le débit liquide et le débit solide. Chaque fichier correspond à une station hydrométrique (cinq stations), sur une période d'observation allant de 1973 à 1995 (tableau 2 et figure 1). 
Tableau 2 Stations hydrométriques du bassin versant de l'oued Mina.

Table 2 Gauging site in the oued Mina basin.

\begin{tabular}{lcrrrc}
\hline $\mathbf{N}^{\circ}$ & Station & $\mathbf{S}\left(\mathbf{k m}^{2}\right)$ & $\mathbf{X}(\mathbf{k m})$ & $\mathbf{Y}(\mathbf{k m})$ & $\mathbf{Z}(\mathbf{m})$ \\
\hline 1 & oued El Abtal & 4126 & 316,80 & 245,65 & 205,00 \\
2 & Sidi A.E.K Djillali & 470 & 309,00 & 244,50 & 225,00 \\
3 & Ain Hamara & 2480 & 316,45 & 235,12 & 300,00 \\
4 & Kef Mehboula & 680 & 331,60 & 224,25 & 475,00 \\
5 & Takhgmaret & 1553 & 316,40 & 203,10 & 600,00 \\
\hline
\end{tabular}

avec S : Surface, $X$ : Longitude, $Y$ : Latitude, $Z$ : Altitude

$X$ et $Y$ coordonnées Lambert Nord d'Algérie.

\subsubsection{Homogénéisation des données pluviométriques}

Afin d'obtenir des séries pluviométriques homogènes (HIEZ, 1977), un comblement de lacunes s'est avéré nécessaire. Le comblement des lacunes annuelles, entre stations, a été fait en utilisant la régression linéaire. Par la suite et afin de combler les séries à l'échelle mensuelle, la méthode des rapports (GHACHI, 1986) a été utilisée. Cette dernière se base sur le rapport entre les mois et les années de la station de base et celle à combler.

$$
\text { Pmij2 = Pmij1 * (Pani2/Pani1) }
$$

Avec Pmij2 : pluie du mois j de l'année i de la station à combler ;

Pmij1 : pluie du mois j de l'année i de la station de base ;

Pani2 : pluie de l'année i de la station à combler ;

Pani1: pluie de l'année i de la station de base.

\section{3 - RÉSULTATS ET DISCUSSION}

\subsection{Variabilité spatio-temporelle de la pluviométrie}

L'étude des variations interannuelles et intersaisonnières des précipitations s'avère essentielle pour connaître l'écoulement saisonnier et le comportement hydrologique annuel d'un bassin versant ( $\mathrm{GHACHI}, 1986)$. En particulier, le régime d'un cours d'eau est en dépendance directe de la pluviométrie, de sa répartition saisonnière et de sa variabilité spatiale et temporelle.

Les précipitations moyennes mensuelles de la période allant de 1973 à 1995 sont représentées à la figure 2. L'analyse de cette figure fait ressortir deux périodes distinctes: une période sèche qui correspond à la saison d'été représentée par les mois de juin, de juillet et d'août, et une période humide qui correspond aux autres mois de l'année.

L'analyse en composantes principales a été employée pour suivre l'évolution temporelle des précipitations. Le but de l'analyse en composantes principales est de transformer un système à $n$ axes en un autre système ayant deux propriétés importantes. La première est que les nouveaux axes, dits principaux, sont orthogonaux, ce qui implique que les projections des observations 
initiales sur chacun de ces nouveaux axes, appelés composantes principales, forment des variables qui sont statistiquement indépendantes. La deuxième propriété est que les composantes principales sont rangées en ordre d'importance décroissante, de sorte que chaque composante explique une quantité maximale de la variance des observations laissées inexpliquées par les composantes précédentes (SIEW-YAN-YU et al., 1998).

L'analyse de cercle de corrélation laisse apparaître trois groupes distincts de stations pluviométriques. Le premier (sud du bassin) est constitué des stations S7, S8, S9 et S10 ; le second (centre du bassin) de S3, S4 et S12. Le troisième groupe (nord du bassin) est formé des stations $\mathbf{S 5}, \mathbf{S 1}, \mathbf{S 2}, \mathbf{S 6}, \mathbf{S 1 5}, \mathbf{S 1 3}$, S11, S14 et S16 (figure 3). La lame d'eau moyenne précipitée dans la partie sud est de $258 \mathrm{~mm}$. Celle du centre est d'environ $291 \mathrm{~mm}$. La partie nord reçoit une pluie moyenne de $300 \mathrm{~mm}$.

L'analyse des projections des variables sur la première composante a permis de déterminer les stations représentatives. Ces stations sont : oued Abtal (oued Mina à la station hydrométrique de oued Abtal), Sidi Abdelkader Djillali (oued El Haddad à Sidi Abdelkader Djillali), Ain Hamara (oued Abd à Ain Hamara), Kef Mehboula (oued Taht à Kef Mehboula) et Ain El Haddid (oued Abd à Takhmaret). L'analyse globale du graphique des projections des observations (années) sur la première composante principale qui explique $51 \%$ de l'information met en évidence deux périodes ; une première excédentaire allant de 1973 à 1980, puis une période déficitaire allant de 1981 jusqu'à 1995 (ACHITE, 1999, ACHITE et TOUAIBIA, 2000 ; figure 4).

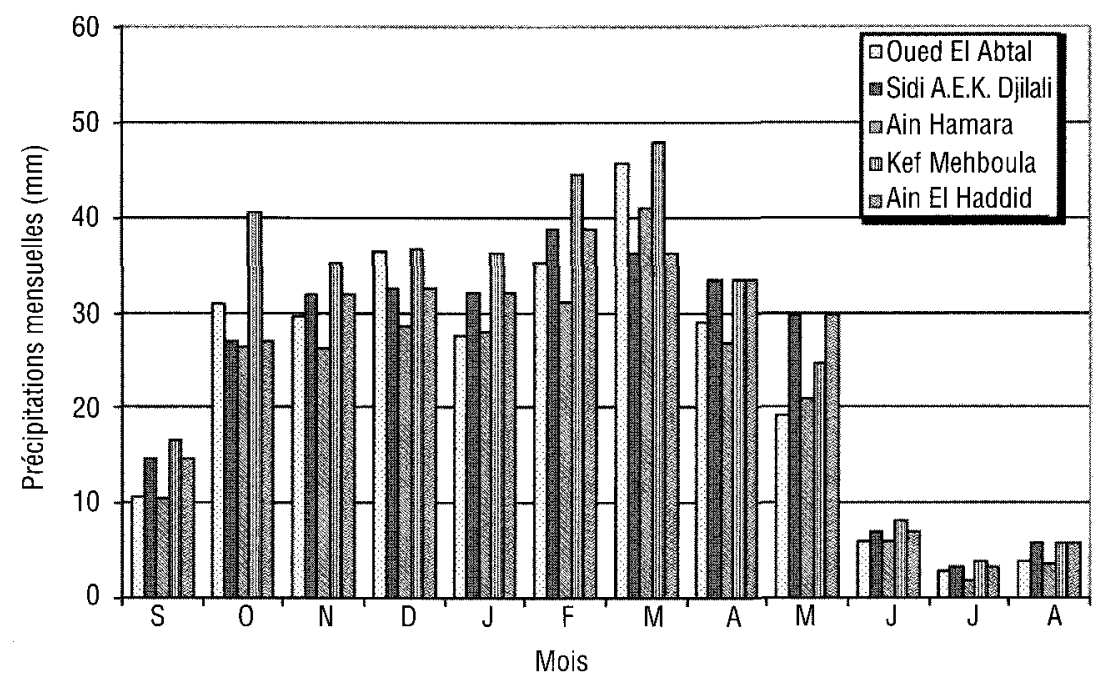

Figure 2 Variabilité spatio-temporelle des pluies mensuelles dans le bassin versant de l'Oued Mina.

Spatial and temporal variation of monthly rainfall in the wadi Mina basin. 


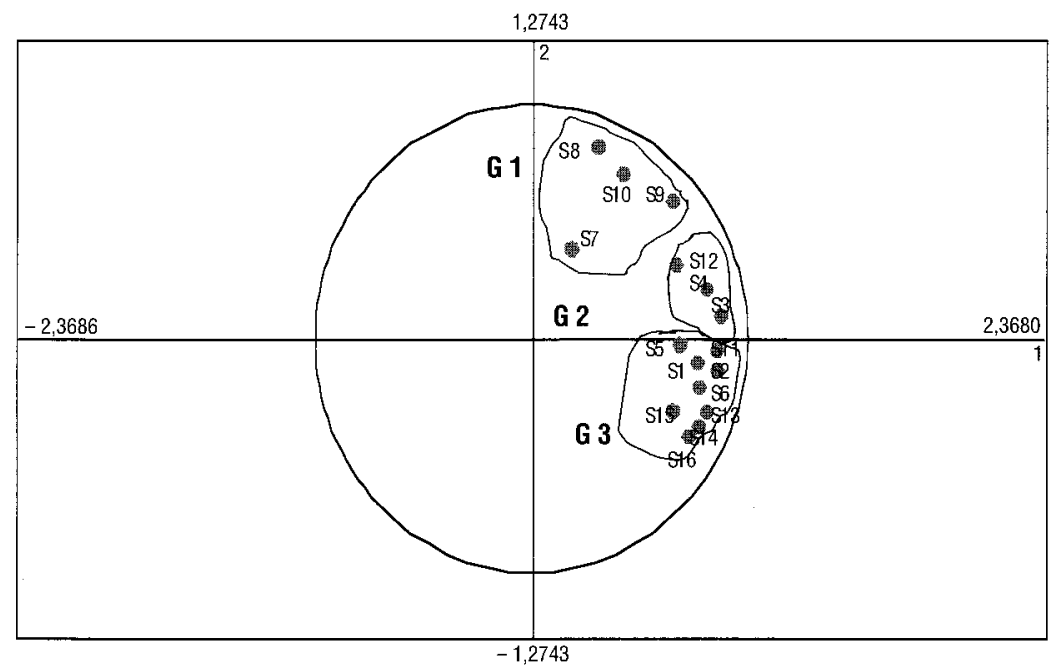

Figure 3 Cercle de corrélation de l'axe 1-2, de l'analyse en composantes principales.

Correlation circle of axe 1-2 of principals components.

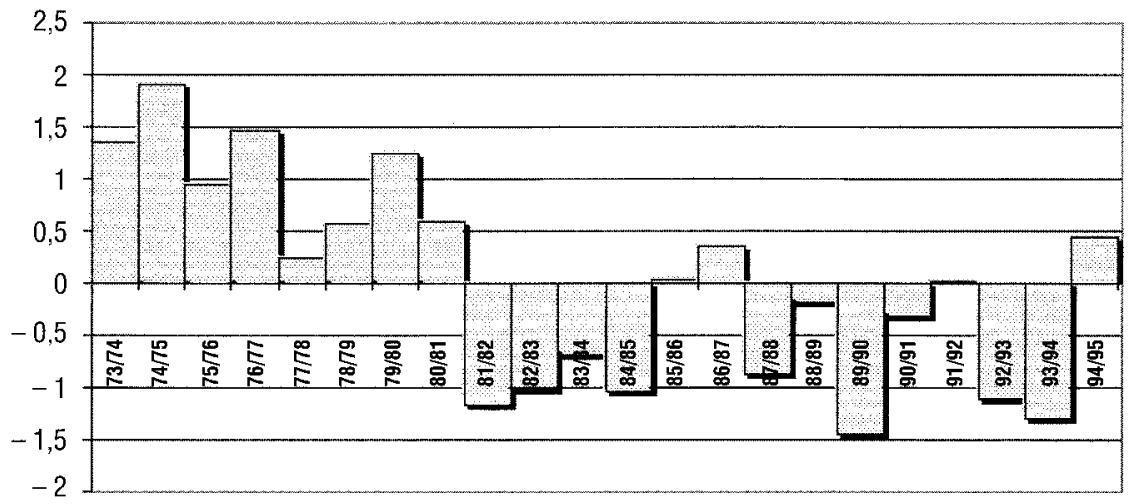

Figure 4 Projection des observations sur la première composante principale [C1].

Projection of observations on the first principal component [C1].

\subsection{Estimation des apports solides par régression}

L'approche méthodologique adoptée consiste à développer un modèle de régression qui permet d'évaluer les valeurs journalières du débit solide à partir des valeurs du débit liquide observées. 
Le tableau 3 présente les coefficients $r^{2}$ pour chacun des modèles de régression. Après plusieurs essais sur les différents types d'ajustement, en se basant sur le coefficient de détermination $r^{2}$ (DAGNELLIE, 1992), le modèle puissance $\left(Q s=a Q_{l}^{b}\right)$ offre le meilleur ajustement. Le modèle retenu (tableau 4) a permis de calculer les apports solides et l'érosion spécifique journalière pour les cinq stations hydrométriques.

Tableau 3 Coefficients de détermination $\left(r^{2}\right)$ des modèles retenus à l'échelle journalière.

Table 3 Determination coefficients $\left(r^{2}\right)$ of selected models at a daily scale.

\begin{tabular}{|c|c|c|c|c|c|}
\hline Stations hydrométriques & 1 & 2 & 3 & 4 & 5 \\
\hline Linéaire & 0,72 & 0,61 & 0,64 & 0,63 & 0,52 \\
\hline Logarithmique & 0,16 & 0,20 & 0,24 & 0,16 & 0,35 \\
\hline Parabolique & 0,72 & 0,70 & 0,80 & 0,79 & 0,69 \\
\hline Puissance* & 0,76 & 0,78 & 0,81 & 0,82 & 0,73 \\
\hline Exponentiel & 0,30 & 0,29 & 0,36 & 0,29 & 0,20 \\
\hline
\end{tabular}

* modèle retenu

Tableau 4 Coefficients de détermination et modèle puissance retenu à l'échelle journalière.

Table 4 Determination coefficient and exponent selected model at a daily scale.

\begin{tabular}{|c|c|c|c|c|c|c|}
\hline \multirow[t]{3}{*}{ Bassins } & \multirow{2}{*}{\multicolumn{3}{|c|}{ 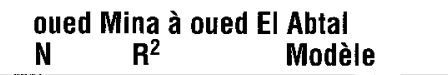 }} & \multirow{2}{*}{\multicolumn{3}{|c|}{ 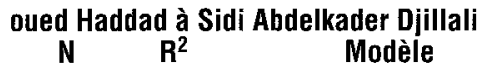 }} \\
\hline & & & & & & \\
\hline & 1080 & 0,76 & $Q s=3,\left.1197 Q\right|^{1,331}$ & 621 & 0,78 & $\mathrm{QS}=9,7338 \mathrm{Q} \mathrm{I}^{1,7141}$ \\
\hline \multirow[t]{3}{*}{ Bassins } & \multicolumn{3}{|c|}{ oued El Abd à Ain Hammara } & \multicolumn{3}{|c|}{ oued El that à Kef Mahboula } \\
\hline & $\mathbf{N}$ & $\mathbf{R}^{2}$ & Modèle & $\mathbf{N}$ & $\mathrm{R}^{2}$ & Modèle \\
\hline & 711 & 0,81 & $Q \mathrm{~S}=1,\left.7411 \mathrm{Q}\right|^{1,7141}$ & 642 & 0,82 & $\mathrm{Qs}=6,531 \mathrm{Q} \mathrm{l}^{1,4632}$ \\
\hline \multirow[t]{2}{*}{ Bassin } & $\mathrm{N}^{\text {ouled }}$ & $\underset{R^{2}}{\text { El Abd }}$ & $\begin{array}{c}\text { Takhmaret } \\
\text { Modèle }\end{array}$ & & & \\
\hline & 421 & 0,73 & $\mathrm{Qs}=4,325 \mathrm{QI}^{1,4112}$ & & & \\
\hline
\end{tabular}

Avec $\mathrm{N}$ : nombre d'observation

Ql : débit liquide

Qs : débit solide

\subsection{Variabilité spatio-temporelle des apports liquide et solide dans le bassin versant de l'oued Mina}

Les transports en suspension de l'oued Mina et de ses affluents présentent des variations spatio-temporelles considérables. Elles sont dues essentiellement au régime pluviométrique, au couvert végétal et à la lithologie. En général, les variations saisonnières des apports solides suivent celles des apports liquides. Les valeurs maximales des apports solides, pour l'ensemble des sous-bassins, s'observent au début d'automne et à la fin du printemps (MEDDI, 1992 et ACHITE, 1999). Généralement, les apports solides minimaux mensuels 
sont enregistrés en été. Cette saison est caractérisée par une faible pluviométrie et par conséquent un faible écoulement superficiel. II est à remarquer cependant que les apports solides minimaux ne correspondent pas forcément au mois caractérisé par le plus faible apport liquide (figure 5). En outre, l'analyse des figures 6 et 7 montre que la saison d'automne contribue en moyenne pour $31 \%$ de l'écoulement liquide annuel et pour $51 \%$ de l'apport solide annuel du bassin versant de l'oued Mina à la station d'oued El Abtal, et avec (26\%, $33 \%),(33 \%, 53 \%),(29 \%, 46 \%)$ et (36\%, $48 \%)$ aux stations de Sidi A.E.K Djillali, Ain Hamara, Kef Mehboula et Takhmaret, respectivement. Le transport solide d'automne reste le plus élevé, dépassant significativement les autres saisons. Cette variabilité s'explique par la variation du couvert végétal (sols nus) durant l'année et la nature agressive des pluies d'automne (RAKOCZI, 1981 et MEDDI, 1992). Ces deux facteurs permettant aux premières crues d'automne de transporter des quantités considérables de sédiments après une longue saison sèche caractérisée par de fortes températures et par la destruction des agrégats du sol par ces dernières. L'énergie produite par les premières pluies ainsi que le faible taux de couverture végétal en cette saison y contribuent.

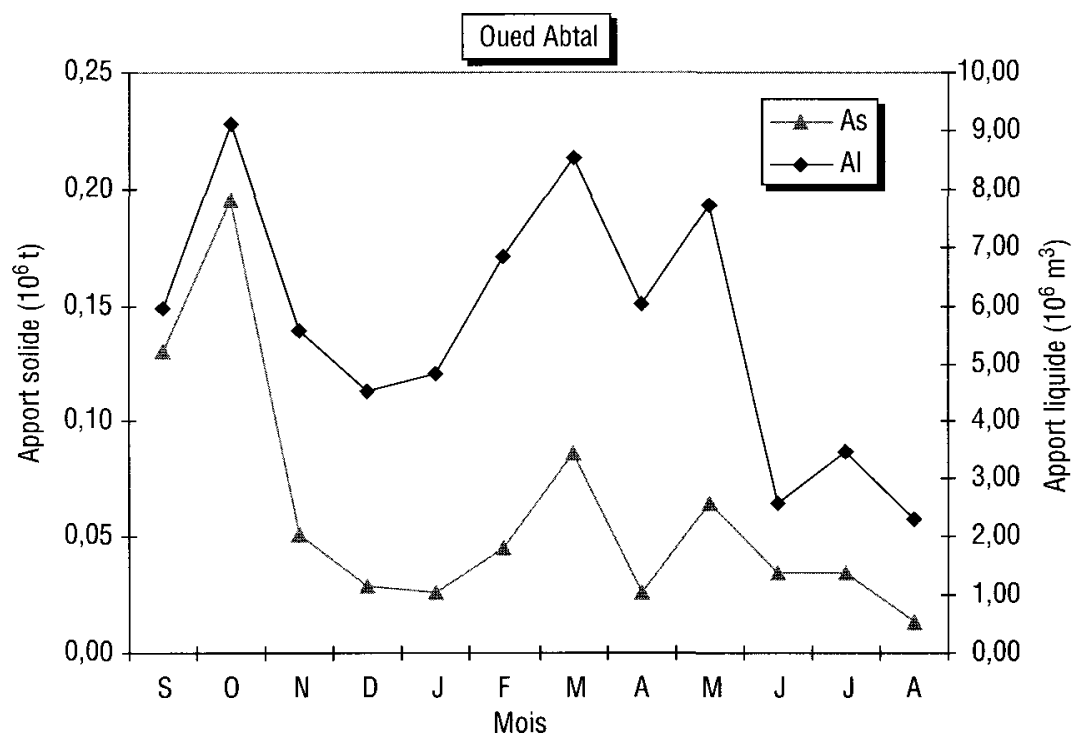

Figure $5 \quad$ Variabilité des apports liquide et solide mensuels dans les sous-bassins versants de l'Oued Mina.

Monthly variability of stream flow and solid yield in the oued Mina basin. 

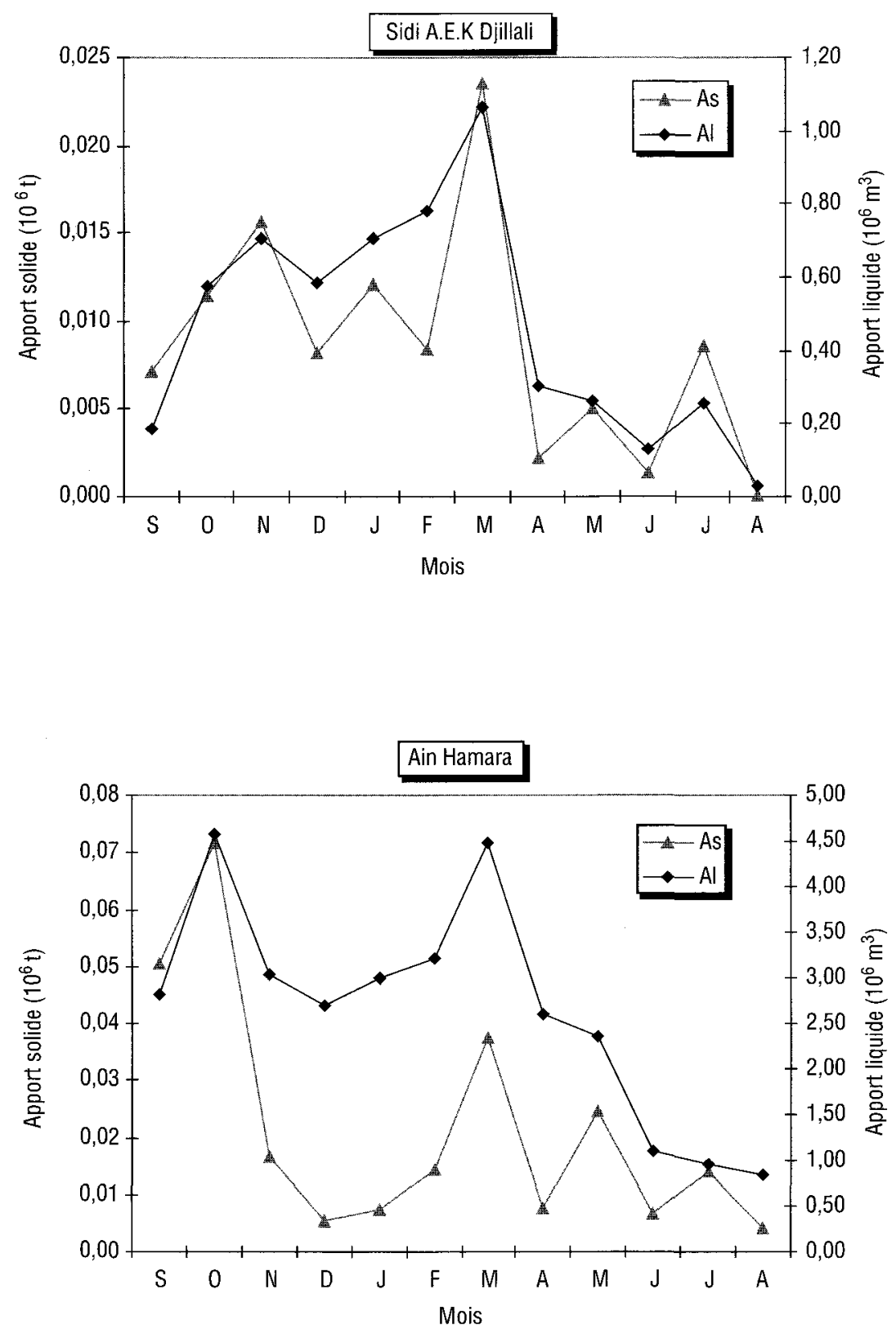

Figure 5 (suite) 

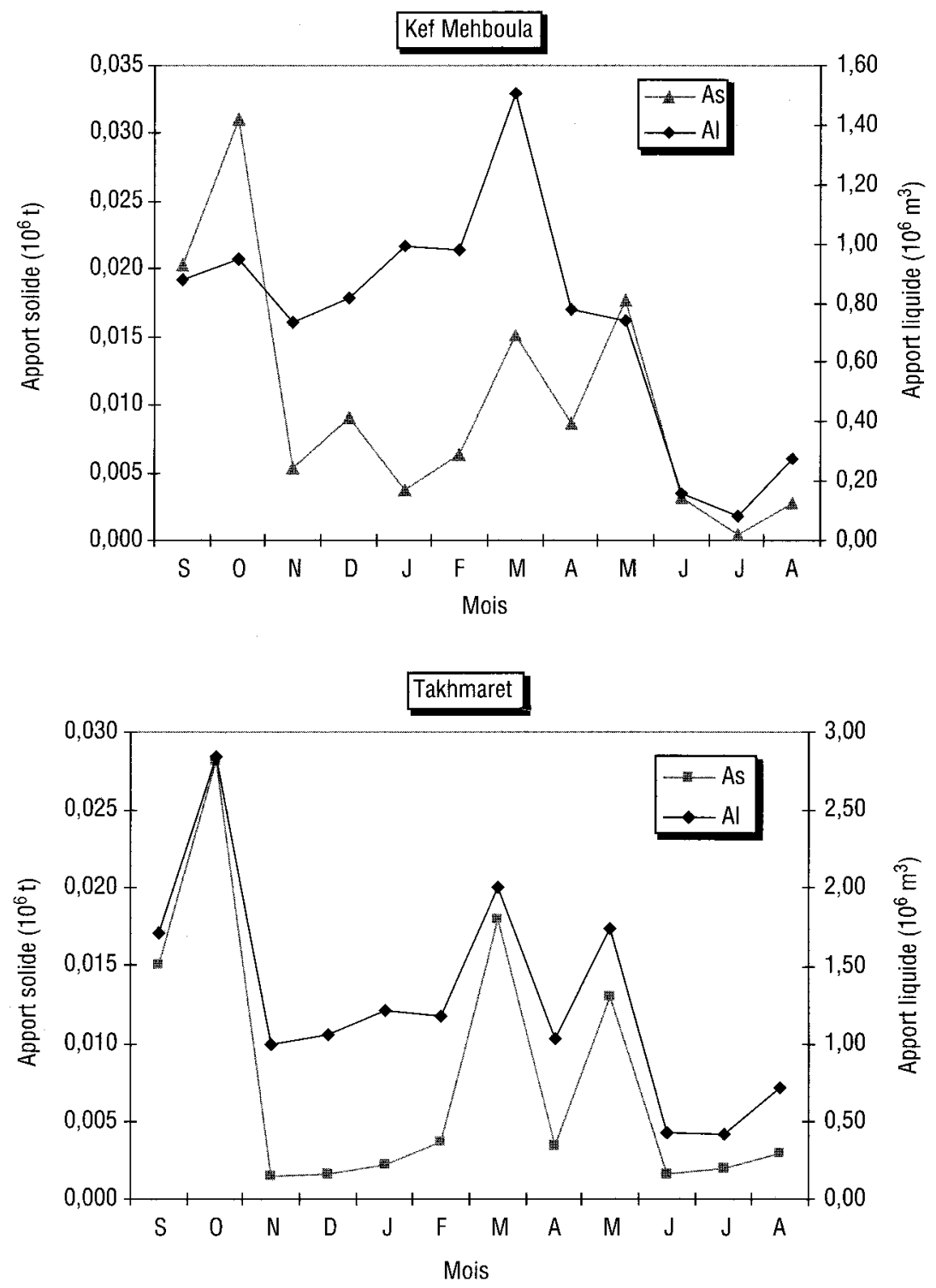

Figure 5 (fin) 


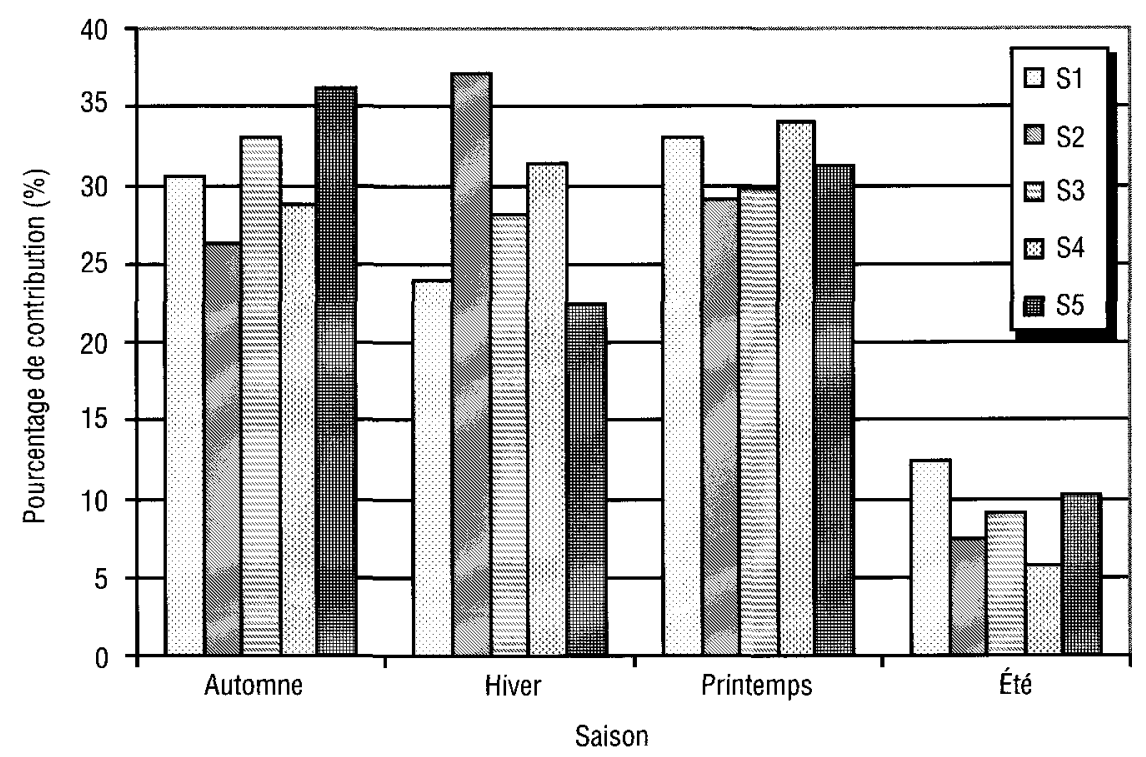

Figure 6 Pourcentage saisonnier des apports liquides.

Seasonal inflow (per cent).

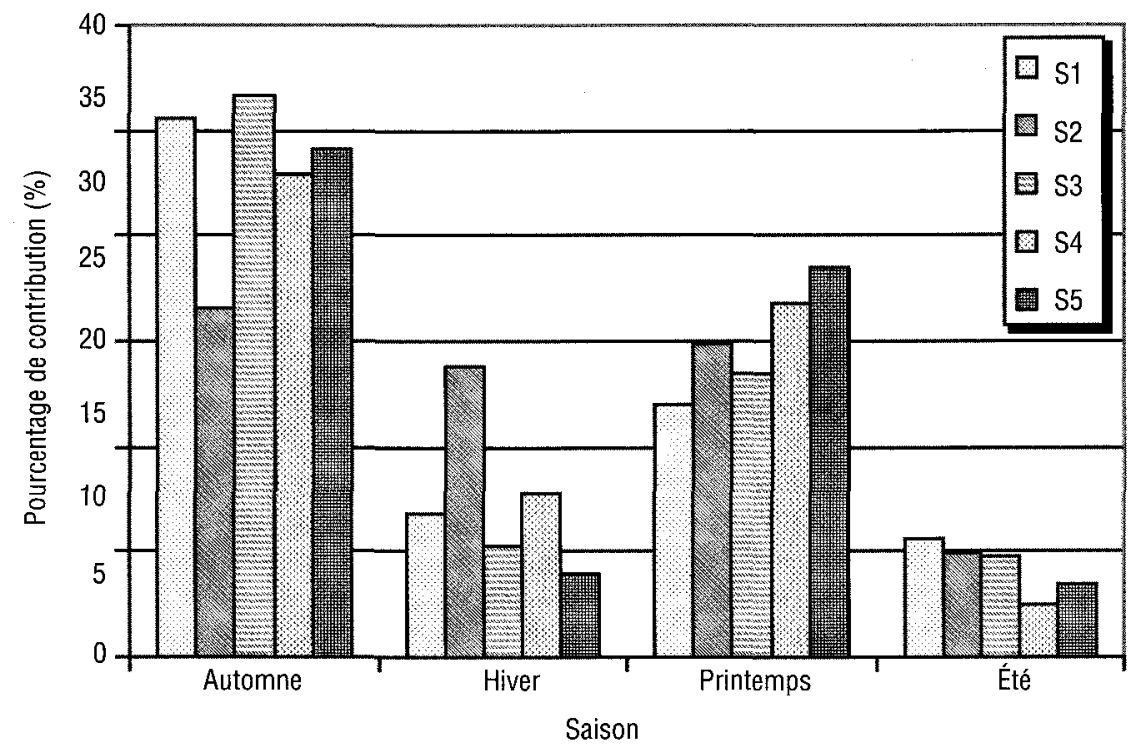

Figure 7 Pourcentage saisonnier des apports solides.

Seasonal sediment yield (per cent). 
À titre de comparaison, la charge spécifique moyenne de l'oued Mina au barrage de Sidi M'hamed Ben Aouda $187 \mathrm{t} / \mathrm{km}^{2} / \mathrm{an}$ (la somme des dégradations de la station d'oued el Abtal et celle de Sidi Abdelkader el Djilali). Elle est supérieure à celle de certains bassins versants faisant partie des régions semi-aride ou sub-aride tels que des bassins versants de la seybousse (Est Algérien, $137 \mathrm{t} / \mathrm{km}^{2} / \mathrm{an}$ ), de l'oued Medjerda (Est Algérien, $113 \mathrm{t} / \mathrm{km}^{2} / \mathrm{an}$ ). Par contre, elle est inférieure à la charge spécifique enregistrée au niveau du bassin versant de la Tafna situé à l'Ouest Algérien 309,9 t/ $\mathrm{km}^{2} / \mathrm{an}$ (la somme des charges spécifiques de la station d'oued Isser, $184 \mathrm{t} / \mathrm{km}^{2} / \mathrm{an}$, et celle d'oued Mouillah, $\left.364 \mathrm{t} / \mathrm{km}^{2} / \mathrm{an}\right)$. La charge spécifique du bassin versant de l'oued Mina est inférieure aux valeurs enregistrées au Maroc où elle varie, pour les bassins versants alimentant 16 barrages, de 240 à $5900 \mathrm{t} / \mathrm{km}^{2} / \mathrm{an}$ (LAHLOU, 1994).

Nous avons constaté que le bassin versant de l'oued Haddad à Sidi Abdelkader el Djilai a une charge spécifique moyenne très élevée par rapport aux autres bassins versants (Ds $=212 \mathrm{t} / \mathrm{km}^{2} / \mathrm{an}$ ). Ce bassin versant est le plus favorable à l'écoulement du point de vue topographique et couverture végétale. II est caractérisé par une couverture végétale discontinue et pauvre en saison chaude. La concentration des crues de la saison chaude au mois d'octobre, et parfois, en mai avec de fortes puissances, donnerait lieu à de fortes concentrations en sédiments durant ces mois. Ce contexte favorable à l'écoulement et par conséquent au transport solide, explique cette forte dégradation des sols dans ce bassin versant (MEDDI, 1992).

Le bassin versant de l'oued Taht, qui draine les hauts plateaux de Frenda, est également favorable à l'écoulement du point de vue topographique et il connaît aussi une pluviométrie élevée par rapport aux autres bassins. Cependant, il enregistre une charge spécifique inférieure à celle du bassin versant de l'oued Haddad (Ds = $191 \mathrm{t} / \mathrm{km}^{2} / \mathrm{an}$ ). Les deux autres bassins versants de l'oued el Abd, à Ain el Hamara et Takhmaret, donnent des valeurs moyennes de l'ordre de $117 \mathrm{t} / \mathrm{km}^{2} /$ an et $65 \mathrm{t} / \mathrm{km}^{2} / \mathrm{an}$ respectivement. Ces valeurs peuvent être expliquées par le caractère moins favorable à l'écoulement des bassins, qui sont caractérisés par des crues de puissance moyenne ou faible (MEDDI, 1992).

À l'échelle inter-annuelle, la variabilité temporelle des apports liquide et solide est remarquable pour certains sous-bassins drainés par les stations hydrométriques (figure 8). En effet, les coefficients de variation (tableau 5) sont très élevés et dépassent même $100 \%$ pour les apports solides des bassins versants de oued Mina à oued El Abtal et de oued Haddad Sidi Abdelkader El Djillali. 


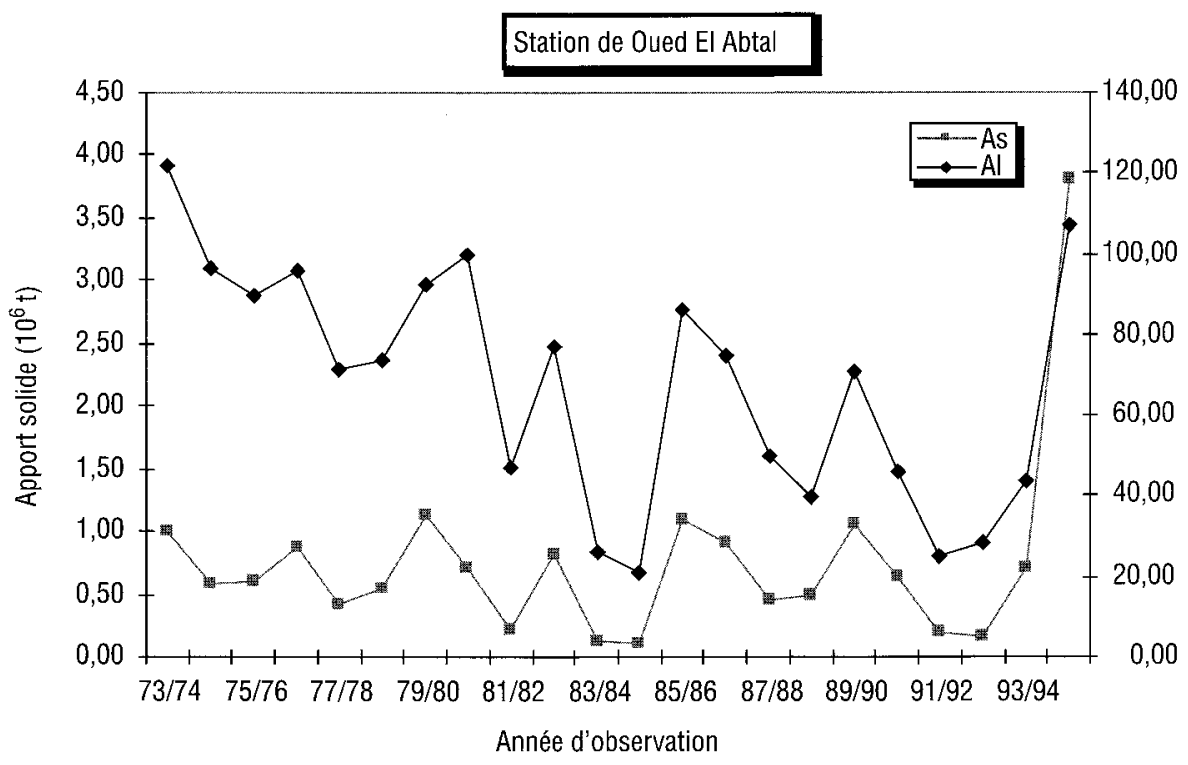

Figure 8 Variabilité interannuelle des apports solide et liquide à la station de Oued Abtal.

Interannual variability of stream flow and solid yields at the Oued El Abtal Gauging site.

Tableau 5 Principaux paramètres statistiques de l'apport liquide (Al) et l'apport solide (As) annuels des sous-bassins drainés par les stations hydrométriques.

Table 5 Principal statistical parameters of the annual flow (Al) and annual solid yield (As) at the sub-basin level.

\begin{tabular}{|c|c|c|c|c|c|c|c|c|c|c|}
\hline \multirow[b]{2}{*}{ Stations } & \multicolumn{2}{|c|}{1} & \multicolumn{2}{|c|}{2} & \multicolumn{2}{|c|}{3} & \multicolumn{2}{|c|}{4} & \multicolumn{2}{|c|}{5} \\
\hline & $\begin{array}{c}A l \\
\left(10^{6} \mathrm{~m}^{3}\right)\end{array}$ & $\begin{array}{c}\text { As } \\
\left(10^{6} t\right)\end{array}$ & $\begin{array}{c}A I \\
\left(10^{6} \mathrm{~m}^{3}\right)\end{array}$ & $\begin{array}{c}\text { As } \\
\left(10^{6} t\right)\end{array}$ & $\underset{\left(10^{6} \mathrm{~m}^{3}\right)}{A I}$ & $\begin{array}{c}\text { As } \\
\left(10^{6} t\right)\end{array}$ & $\begin{array}{c}\text { Al } \\
\left(10^{6} \mathrm{~m}^{3}\right)\end{array}$ & $\begin{array}{c}\text { As } \\
\left(10^{6} t\right)\end{array}$ & $\underset{\left(10^{6} \mathrm{~m}^{3}\right)}{A l}$ & $\begin{array}{c}\text { As } \\
\left(10^{6} t\right)\end{array}$ \\
\hline Max. & 122,03 & 3,81 & 10,79 & 0,39 & 44,69 & 1,44 & 18,35 & 0,60 & 36,69 & 0,63 \\
\hline Min. & 20,74 & 0,11 & 0,78 & 0,1 & 0,97 & 0,03 & 1,76 & 0,003 & 1,36 & 0,01 \\
\hline Moy. & 67,41 & 0,76 & 5,42 & 0,10 & 18,71 & 0,29 & 9,44 & 0,13 & 14,04 & 0,10 \\
\hline Écart Type & 29,78 & 0,75 & 2,77 & 0,09 & 15,11 & 0,36 & 4,78 & 0,15 & 10,30 & 0,15 \\
\hline C.V $(\%)$ & 44 & 101 & 51 & 113 & 81 & 80 & 51 & 86 & 73 & 62 \\
\hline
\end{tabular}

\subsection{Modélisation du transport solide à l'aide du coefficient de ruissellement}

Après avoir développé un modèle liant le transport solide au débit liquide, il nous a paru judicieux de mettre au point un modèle explicatif du transport solide en fonction des paramètres conditionnant ce phénomène. Le coefficient de ruissellement $(\mathrm{Ce}$ ) dépend des facteurs suivants : la pluviométrie, la nature du sol, le relief, la géologie et le couvert végétal. Ces derniers peuvent égale- 
ment être considérés comme des facteurs explicatifs du transport solide. À partir de cette démarche et étant donné la disponibilité des données concernant le coefficient de ruissellement $(\mathrm{Ce})$, un modèle puissance de type As = aCeb a été développé pour chaque sous-bassin (tableau 6). La figure 9 présente, à titre d'exemple, la relation trouvée pour le bassin versant de l'oued Mina à la station d'oued El Abtal.

Pour développer les modèles, deux tiers des données ont été utilisés et le tiers restant a été pris comme échantillon témoin afin de valider les résultats. Les critères basés sur la minimisation des écarts entre valeurs observées et calculées (MEDDI, 1992 ; SPIEGEL, 1989) ont été utilisés. Ces critères sont le coefficient de détermination $\left(r^{2}\right)$, le test de Fischer $F_{c}$, et l'erreur type $E_{y, x}$. Les modèles développés et les paramètres de validation sont résumés dans le tableau 6. Le coefficient d'écoulement (Ce) explique plus de $60 \%$ de la variance du transport solide pour les cinq bassins versants étudiés. La variance non expliquée peut être traduite par les apports dus aux sapements des berges des cours d'eau, par l'état du sol, par les pratiques culturales et par les erreurs dues aux mesures. Les erreurs trouvées, après validation sur le tiers des données, sont de l'ordre de $15 \%$.

Tableau 6 Paramètres de validation et modèles retenus pour les différents sousbassins.

Table 6 Validation parameters and developed models for different sub-basins.

\begin{tabular}{|c|c|c|c|c|c|}
\hline Stations & $\mathrm{N}$ & $\mathbf{R}^{2}$ & $E_{x-y}$ & Fc & modèles \\
\hline oued El Abtal & 15 & 0,79 & 0,15 & 49,33 & $A s=0,0453 C \mathrm{e}^{1,3152}$ \\
\hline Sidi A.E.K Djillali & 15 & 0,61 & 0,16 & 20,58 & As $=0,013 C \mathrm{e}^{1,0879}$ \\
\hline Ain Hamara & 15 & 0,64 & 0,12 & 23,23 & $A s=0,0203 C \mathrm{e}^{1,157}$ \\
\hline Kef Mehboula & 15 & 0,78 & 0,20 & 47,09 & As $=0,0064 C \mathrm{e}^{1,7296}$ \\
\hline Takhmaret & 15 & 0,65 & 0,27 & 24,46 & As $=0,0038 C \mathrm{e}^{2,0082}$ \\
\hline
\end{tabular}

Avec $\mathrm{N}$ : nombre d'observation, $\mathrm{R}^{2}$ : coefficient de détermination, $\mathrm{E}_{\mathrm{x}-\mathrm{y}}$ : erreur type, $\mathrm{Fc}_{\mathrm{c}}$ : test de Fischer.

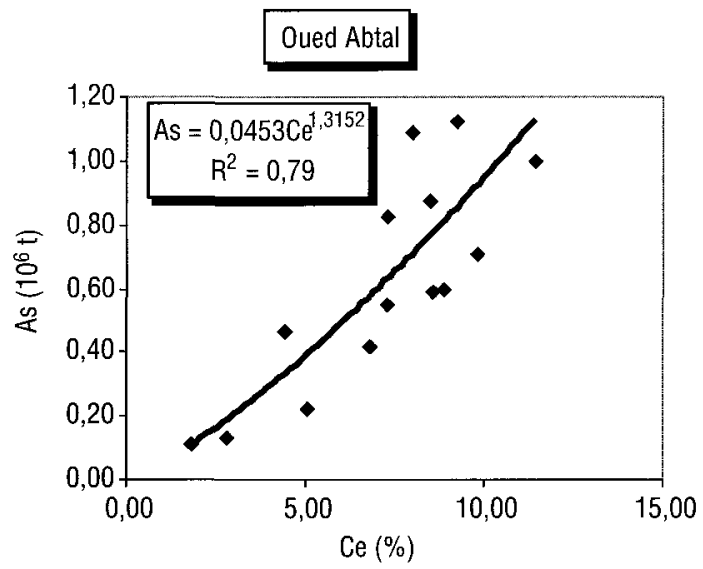

Figure 9 Relation apport solide - coefficient d'écoulement à la station de Oued El Abtal.

Relation between sediment yield and runoff coefficient in the Oued Mina basin at the Oued El Abtal gauging site. 


\section{4 - CONCLUSION}

L'érosion hydrique des sols constitue un aspect majeur de la dégradation des paysages dans les environnements méditerranéens. Au cours de cette étude, il a été possible de définir une approche statistique d'évaluation des apports de solides en suspension basée sur les relations débit liquide - débit solide. Cette approche globale semble satisfaisante pour la quantification des apports solides dans les bassins versants jaugés à partir des données observées de débit liquide et de concentration en matière en suspension.

La variation du taux des apports solides et liquides entre stations peut être expliquée, d'une part, par le fait que les sous-bassins étudiés présentent une variabilité dans le régime d'écoulement (variabilité spatio-temporelle des précipitations) et d'autre part, par la variabilité des caractéristiques hydromorphométriques. L'essentiel du transfert des matières en suspension se produit au cours de crues violentes qui surviennent généralement en automne.

Le bassin versant de l'oued Haddad présente une charge spécifique élevée par rapport aux autres bassins (Ds $=212 \mathrm{t} / \mathrm{km}^{2} / \mathrm{an}$ ). Cette dégradation des sols est très visible sur le terrain. Elle est due aux caractères favorables à l'érosion : (i) une topographie très favorable (pentes raides), qui conduit à un écoulement très rapide qui favorise l'arrachement des particules du sol après le travail de désagrégation par insolation; et (jj) une couverture végétale discontinue, qui protège mal le sol. Le bassin versant de l'oued Taht, qui a une topographie favorable à l'écoulement et des pluies abondantes, a une charge spécifique inférieure à celle du bassin précédent ( $\mathrm{Ds}=191 \mathrm{t} / \mathrm{km}^{2} / \mathrm{an}$ ). Les bassins versants de l'oued el Abd présentent des charges spécifiques de l'ordre de $117 \mathrm{t} / \mathrm{km}^{2} / \mathrm{an}$ à Ain el Hamara et de $65 \mathrm{t} / \mathrm{km}^{2} / \mathrm{an}$ à Takhmaret. L'oued Mina au barrage de Sidi M'hamed Ben Aouda donne une charge spécifique de $187 \mathrm{t} / \mathrm{km}^{2} / \mathrm{an}$.

Le modèle puissance traduit bien la relation entre le transport solide et le coefficient de ruissellement, pris comme facteur explicatif, car plus de $60 \%$ de la variance est expliquée par ce dernier. Le taux de la variance expliquée par le coefficient de ruissellement est inférieur à celui expliqué par le débit liquide dans le premier modèle. Donc, cette approche ne donne pas de meilleurs résultats pour le bassin étudié.

Les modèles développés peuvent être utilisés pour la prévision du transport solide. Les mesures et la prévision de la concentration en matière en suspension revêtent un grand intérêt, car elles permettent une quantification fiable des apports solides aux différentes stations. Elles permettent ainsi de classer les sous-bassins d'un bassin versant en terme de contribution à l'envasement du barrage de Sidi M'hamed Ben Aouda et de classer les zones par ordre de priorités en matière d'aménagement anti-érosif. 


\section{RÉFÉRENCES BIBLIOGRAPHIQUES}

ACHITE M., 1999. Analyse multivariée de la variable «Érosion Spécifique »: cas du bassin versant de l'oued Mina. Thèse de Magister, École Nationale Supérieure d'Hydraulique, Blida, Algérie, 206 p.

ACHITE M., TOUAIBIA B., 2000. Analyse multivariée de la variable «Érosion Spécifique ": cas du bassin versant de l'oued Mina (Algérie). Séminaire International d'hydrologie des zones méditerranéennes. Montpellier, France.

ACHITE M. 2002. Approche statistique d'évaluation du transport solide dans le bassin versant de l'oued Mina (nord ouest Algérien). Colloque international sur l'eau dans le bassin méditerranéen: Ressources et développement durable, 10-13 octobre 2002, Monastir (Tunisie), pp. 894-899.

BENKHALED A., REMINI B., 2003. Analyse de la relation de puissance: débit solide - débit liquide à l'échelle du bassin versant de l'oued Wahrane (Algérie). Rev. Sci. Eau, 16, 333-356.

BERGAOUI M., CAMUS H., NOUVELOT J.F., 1998. Essai de modélisation du transport solide sur les microbassins versants de Tebaga (Tunisie Centrale), Cahier sécheresse, $9(1), 51-57$.

BOU KHEIR R., GIRARD M-CI., KHAWLIE M. et ABADALLAH C. (2001). Érosion hydrique des sols dans les milieux méditerranéens: une revue bibliographique. Étude et gestion des sols, 8(4), 231-245.

DAGNELLIE P., 1992. Théorie et méthodes statistiques. Applications agronomiques. Presses agronomiques de Gembloux, Belgique, $463 \mathrm{p}$.

DELHOUME J-P., 1981. Études en milieu méditerranéen semi-aride : ruissellement et érosion en zone montagne de Tunisie centrale (djebel Semmama). Campagnes 1975 à 1979. Tunis, Orstom/DRE, 187 p.

DEMMAK A., 1982. Contribution à l'étude de l'érosion et des transports solides en Algérie septentrionale. Thèse doct. Ing. Paris, ; $323 \mathrm{p}$.

DEMMAK A., 1984. Recherche d'une relation empirique entre les apports solides spécifiques et les paramètres physico climatiques des bassins : cas algérien. In. AISH Public 144 : 403-414.
DUBREUIL P., GUISCAFRE J., 1971. La planification du réseau hydrométrique minimal. Cahiers ORSTOM, Série Hydrologie, vol. VIII, $n^{\circ} 2,3-38$.

FERGUSON R.I., 1987. Accuracy and precision of methods for estimating river loads. Earth Surface Processes and Landforms, 12, 95-104.

GHACHI A., 1986. Hydrologie et utilisation de la ressource en eau: Bassin de la Seybouse. Office des publications universitaires (OPU), Alger, 508 p.

HEUSCH B., 1970. L'érosion du Pré-Rif, annales rech. Forestière, Maroc, 12, $176 \mathrm{p}$.

HIEZ G., 1977. L'homogénéité des données pluviométriques. Cahiers ORSTOM, Série Hydrologie, vol XIV, $\mathrm{n}^{\circ} 2,129-172$.

KASSOUL A. ABDELGADER A. et BELORGEY M., 1997. Caractérisation de la sédimentation des barrages en Algérie. Rev. Sci. Eau, 10/3, 339-358.

KOURI L., 1993. L'érosion hydrique des sols dans le bassin versant de l'oued Mina. Étude des processus et type de fonctionnement des ravins dans la zone des marnes tertiaires. Thèse de doctorat. Univ. Louis Pasteur, Strasbourg, France, 238 p.

LAHLOU A., 1994 Envasement des barrages au Maroc. Édition WALLADA, Casablanca, Maroc, $277 \mathrm{p}$.

MAHIEDDINE M., 1997. Quantification et variabilité parcellaire sous simulation de pluie dans le bassin versant de l'oued Mina. Thèse de Magister. École Nationale Supérieure des Sciences Agronomiques, Alger, $120 \mathrm{p}$.

MEDDI M., 1992. Hydro-pluviométrie et transport solide dans le bassin versant de l'oued Mina (Algérie). Thèse de doctorat, Univ. Louis Pasteur, Strasbourg, France, $285 \mathrm{p}$.

MEDDI M., KHALDI A., MEDDI H., 1998. Étude du transport solide dans le nord de I'Algérie. IAHS Publication. Proceedings, International Symposium « Modelling Soil Erosion, Sediment Transport and Closely Related Hydrological Processes ", Vienna, pp. 393-397.

MEDDI M., 1999. Étude du transport solide dans le bassin versant de l'oued Ebda 
(Algérie). Zeitschrift fûr Geomorphologie, $43,167-183$

MEDDI M., MORSLI B., 2001. Étude d'érosion et du ruissellemnt sur bassins versants expérimentaux dans les monts de Beni-Chougrane (Ouest d'Algérie). Zeitschrift für Geomorphologie, 45, 443-452.

RAKOCZI L., 1981. Problems of sediment gauging in gravel-beds, $\mathrm{XIX}$ congress, New Delhi, India., pp 105-110

SERRAT P., LUDWIG W., NAVARRO B., BLAZI J., 2001. Variabilité spatio-temporelle des flux des matières en suspension d'un fleuve méditerranéen: la têt (France), C.R. Acad. Sci. Paris, Science de la terre et des planètes, 333, 389397.

SIEW-YAN-YU T.O., ROUSSELLE J., JACQUES G., NGUYEN V.T.V., 1998. Régionalisation du régime des précipitations dans la région des Bois-francs et de l'Estrie par l'analyse en composantes principales. Can. J. Civ. Eng., 25, 10501058.
SPIEGEL M.R., 1989. Probabilités et statistiques - cours et problèmes - Série Shaum, McGraw Hill, Paris, 385 p.

TERFOUS, A., MEGHNOUFI, A., BOUANANI, A., 2001. Étude du transport solide en suspension dans l'oued Mouilah (Nord Ouest Algérien). Rev. Sci. Eau, $14: 173-185$.

WALLING D. E. \& WEBB D. W., 1981. The reliability of suspended sediment load data: Erosion and sediment transport measurement, Proceedings of the Florence Symposium, Florence: IAHS publ.133, pp. 177-194.

WALLING D. E. and WEBB B. W., 1988. The reliability of rating curve estimates of suspended sediment yield: Some further comments, sediment budgets. Symposium on Sediment Budgets, Porto Alegre, Brazil: IAHS publ. 174, pp. 337350. 